\title{
A TREE-FREE GROUP THAT IS NOT ORDERABLE
}

\author{
SHANE O ROURKE
}

(Communicated by Kevin Whyte)

\begin{abstract}
I. M. Chiswell has asked whether every group that admits a free isometric action (without inversions) on a $\Lambda$-tree is orderable. We give an example of a multiple HNN extension $\Gamma$ which acts freely on a $\mathbb{Z}^{2}$-tree but which has non-trivial generalised torsion elements. The existence of such elements implies that $\Gamma$ is not orderable.
\end{abstract}

Let $\Lambda$ be an ordered abelian group. A group is $\Lambda$-free if it admits a free isometric action without inversions on a $\Lambda$-tree, and tree-free if it is $\Lambda^{\prime}$-free for some $\Lambda^{\prime}$. We refer to the book [4] for a detailed account of the fundamentals of $\Lambda$-trees.

In this book Chiswell asks [4, §5.5, Question 3] whether all tree-free groups are orderable, or at least right-orderable. (This question also appeared in his earlier paper [3.) There has recently been some progress made on questions of orderability in tree-free groups. Chiswell himself has shown [6. Theorem 3.8] that $\mathbb{R}^{n}$-free groups are right-orderable. Kharlampovich, Myasnikov and Serbin have shown [10, Corollary 4] that finitely presented tree-free groups are $\mathbb{R}^{n}$-free for some $n$; thus these groups are right-orderable. Chiswell has shown moreover [5, Theorem 4.5] that tree-free groups admit a locally invariant order.

In their recent survey Kharlampovich, Myasnikov and Serbin state [11, Corollary 19] that finitely presented tree-free groups have a finite index subgroup that embeds in a right-angled Artin group: this is a consequence of their result [10, Theorem 2] and the extensive work of Wise (see [16, §16] and [15]) on quasi-convex hierarchies on groups. Since right-angled Artin groups are residually torsion-free nilpotent (see [8, Chapter 3, Theorem 1.1]), it follows that finitely presented treefree groups are virtually residually torsion-free nilpotent; hence they are virtually orderable.

Let us also note that tree-free surface groups admit an embedding in a rightangled Artin group (see [7, Theorem 3]); it follows, using Rips' Theorem (see [4, Chapter 6]), that finitely generated $\mathbb{R}$-free groups admit an embedding in a rightangled Artin group.

The author has recently 13 raised the question of whether $\mathbb{Z}^{n}$-free groups are residually torsion-free nilpotent. An affirmative answer to this question would have implied that $\mathbb{Z}^{n}$-free groups are orderable.

Nevertheless the answer to Chiswell's question is negative, even when restricted to finitely presented $\mathbb{Z}^{2}$-free groups as we will show presently. It follows that the

Received by the editors November 29, 2012 and, in revised form, March 1, 2013.

2010 Mathematics Subject Classification. Primary 20E08; Secondary 20F60.

The author would like to thank Ian Chiswell for helpful conversations. 
word 'virtually' cannot be dropped in the discussion above. This suggests an analogy with the situation of braid groups $B_{n}$ and their finite index subgroups, the pure braid groups $P_{n}$ : the former are right-orderable but not orderable (see [14, §4]), while the latter are residually torsion-free nilpotent [9]. Moreover finitely presented tree-free groups, like braid groups, are now known to be linear: see [11, Theorem 68], 2 and 12 .

Recall that a group $G$ is orderable if there is a linear order $\leq$ on $G$ satisfying $x \leq y \Rightarrow g x h \leq g y h$ for $g, h \in G$. (One can define right-orderable by restricting to $g=1$.) It is well-known and easy to see that in an orderable group $G$ there can be no non-trivial generalised torsion elements: these are elements $g$ such that $g^{h_{1}} g^{h_{2}} \cdots g^{h_{n}}=1$ for some $h_{1}, \ldots, h_{n} \in G$ and $n \geq 1$. (Here $g^{h}$ denotes the conjugate $\left.h^{-1} g h.\right)$

Let $F$ be the free group on $\{x, y, z\}$, and consider the natural free action of $F$ on the corresponding Cayley graph, viewed as a $\mathbb{Z}$-tree. Observe that $x y^{-1}, y z^{-1}$ and $z x^{-1}$ and their respective inverses belong to distinct conjugacy classes since they are cyclically reduced as elements of $F$ and none is a cyclic permutation of another. Moreover, the translation length of $g \in F$ is equal to the word length of a cyclically reduced conjugate of $g$. Thus the translation lengths of $x y^{-1}, y z^{-1}$ and $z x^{-1}$ are all equal to 2 .

Now taking $s_{1}=x y^{-1}=s_{2}, t_{1}=y z^{-1}, t_{2}=z x^{-1}, u=u_{1}$ and $v=u_{2}$, and applying [1, Proposition 4.19], the multiple HNN extension

$$
\begin{aligned}
\Gamma & =\left\langle x, y, z, u_{1}, u_{2} \mid u_{1} s_{1} u_{1}^{-1}=t_{1}, u_{2} s_{2} u_{2}^{-1}=t_{2}\right\rangle \\
& =\left\langle u, v, x, y, z \mid u\left(x y^{-1}\right) u^{-1}=y z^{-1}, v\left(x y^{-1}\right) v^{-1}=z x^{-1}\right\rangle
\end{aligned}
$$

is seen to be $\mathbb{Z}^{2}$-free. However,

$$
1=\left(x y^{-1}\right)\left(y z^{-1}\right)\left(z x^{-1}\right)=x y^{-1} \cdot u\left(x y^{-1}\right) u^{-1} \cdot v\left(x y^{-1}\right) v^{-1},
$$

whence $x y^{-1}$ is a non-trivial generalised torsion element of $\Gamma$, and $\Gamma$ is not orderable. This gives the promised negative answer to Chiswell's question.

\section{REFERENCES}

[1] Hyman Bass, Group actions on non-Archimedean trees, Arboreal group theory (Berkeley, CA, 1988), Math. Sci. Res. Inst. Publ., vol. 19, Springer, New York, 1991, pp. 69-131, DOI 10.1007/978-1-4612-3142-4_3. MR1105330 (93d:57003)

[2] Stephen J. Bigelow, Braid groups are linear, J. Amer. Math. Soc. 14 (2001), no. 2, 471-486 (electronic), DOI 10.1090/S0894-0347-00-00361-1. MR1815219(2002a:20043)

[3] I. M. Chiswell, Non-standard free groups, Model theory of groups and automorphism groups (Blaubeuren, 1995), London Math. Soc. Lecture Note Ser., vol. 244, Cambridge Univ. Press, Cambridge, 1997, pp. 153-165, DOI 10.1017/CBO9780511629174.011. MR.1689867 (2000f:03110)

[4] Ian Chiswell, Introduction to $\Lambda$-trees, World Scientific Publishing Co. Inc., River Edge, NJ, 2001. MR:1851337(2003e:20029)

[5] I. M. Chiswell, Locally invariant orders on groups, Internat. J. Algebra Comput. 16 (2006), no. 6, 1161-1179, DOI 10.1142/S0218196706003463. MR.2286427 (2007k:20083)

[6] I. M. Chiswell, Right orderability and graphs of groups, J. Group Theory 14 (2011), no. 4, 589-601, DOI 10.1515/JGT.2010.065. MR.2818951 (2012f:20084)

[7] John Crisp and Bert Wiest, Embeddings of graph braid and surface groups in rightangled Artin groups and braid groups, Algebr. Geom. Topol. 4 (2004), 439-472, DOI 10.2140/agt.2004.4.439. MR2077673 (2005e:20052)

[8] C. Droms, Graph groups, Ph.D. thesis, Syracuse University, 1983, available from http://educ.jmu.edu/dromscg/vita/thesis/. 
[9] Michael Falk and Richard Randell, Pure braid groups and products of free groups, Braids (Santa Cruz, CA, 1986), Contemp. Math., vol. 78, Amer. Math. Soc., Providence, RI, 1988, pp. 217-228, DOI 10.1090/conm/078/975081. MR.975081 (90d:20070)

[10] O. Kharlampovich, A. Myasnikov, and D. Serbin, Groups acting freely on $\Lambda$-trees, arXiv:0911.0209v4, November 2011.

[11] Actions, length functions, and non-archimedean words, Internat. J. Algebra 23 (2013), no. 2, 325-455. MR3038860

[12] Daan Krammer, Braid groups are linear, Ann. of Math. (2) 155 (2002), no. 1, 131-156, DOI 10.2307/3062152. MR.1888796(2003c:20040)

[13] Shane O Rourke, Affine actions on non-Archimedean trees, Internat. J. Algebra Comput. 23 (2013), no. 2, 217-253. MR.3038858

[14] Dale Rolfsen, New developments in the theory of Artin's braid groups, Proceedings of the Pacific Institute for the Mathematical Sciences Workshop "Invariants of Three-Manifolds" (Calgary, AB, 1999), 2003, pp. 77-90, DOI 10.1016/S0166-8641(02)00054-8. MR.1953321 (2004f:20070)

[15] Daniel T. Wise, Research announcement: the structure of groups with a quasiconvex hierarchy, Electron. Res. Announc. Math. Sci. 16 (2009), 44-55, DOI 10.3934/era.2009.16.44. MR2558631(2011c:20052)

[16] Daniel T. Wise, The structure of groups with a quasiconvex hierarchy, available from http://www.math.mcgill.ca/wise/papers.html, 2011.

Department of Mathematics, Cork Institute of Technology, Rossa Avenue, Cork, IRELAND

E-mail address: shane.orourke@cit.ie 